Article

\title{
Targeted Analysis of 46 Bile Acids to Study the Effect of Acetaminophen in Rat by LC-MS/MS
}

\author{
Vivaldy Prinville, Leanne Ohlund and Lekha Sleno *(D) \\ Department of Chemistry, Université du Québec à Montréal (UQAM), P.O. Box 8888 Downtown Station, \\ Montreal, QC H3C 3P8, Canada; vivaldyp@hotmail.com (V.P.); ohlund@gmail.com (L.O.) \\ * Correspondence: sleno.lekha@uqam.ca
}

Received: 30 September 2019; Accepted: 6 January 2020; Published: 7 January 2020

\begin{abstract}
Bile acids represent a large class of steroid acids synthesized in the liver and further metabolized by many bacterial and mammalian enzymes. Variations in bile acid levels can be used as a measure of liver function. There still exists, however, a need to study the variation of individual circulating bile acids in the context of hepatotoxity or liver disease. Acetaminophen (APAP), a drug commonly taken to relieve pain and decrease fever, is known to cause acute liver failure at high doses. We have developed a targeted liquid chromatography-tandem mass spectrometry method to monitor the effects of different doses of APAP on the bile acid plasma profile in a rat model. The analysis method was optimized to ensure chromatographic resolution of isomeric species using a mixture of 46 standard bile acids, and 14 isotopically-labeled internal standard (IS) compounds detected in multiple reaction monitoring (MRM) mode on a triple quadrupole mass spectrometer. Four doses of acetaminophen were studied, the highest of which shows signs of hepatotoxicity in rats. This targeted method revealed that high dose APAP has an important effect on bile acid profiles. Changes were seen in several unconjugated bile acids as well as glycine conjugates; however, no obvious changes were apparent for taurine-conjugated species.
\end{abstract}

Keywords: bile acids; metabolomics; rat plasma; tandem mass spectrometry; liquid chromatography; acetaminophen; hepatotoxicity

\section{Introduction}

Bile acids play many roles crucial for metabolism and liver health. They are formed from cholesterol through a series of enzymatic reactions and they represent the primary pathway for cholesterol catabolism [1]. In addition, bile acids emulsify fat from our diet and help absorb lipids and cholesterol [2]. Primary bile acids, such as cholic acid (CA) and chenodeoxycholic acid (CDCA) in humans and $\alpha$-muricholic acid ( $\alpha$-MCA) and $\beta$-muricholic acid ( $\beta$-MCA) in rodents, are synthesized in the liver. Before being secreted by the liver, bile acids can be conjugated to taurine or glycine amino acids. In the intestines, bile acids are unconjugated and converted into secondary bile acids, such as deoxycholic acid (DCA) and lithocholic acid. Most bile acids are reabsorbed in the liver, conjugated again, and excreted in the bile to complete the enterohepatic circulation [2,3].

An increased plasma concentration of bile acids is a sign of liver disease [4]. High concentrations are toxic, though the potential for toxicity depends on the bile acid profile. For example, it has been reported that chenodeoxycholic acid and lithocholic acid, as well as their conjugates, can damage hepatic cells and induce mitochondrial malfunction, oxidative stress, and apoptosis [5-8]. Bile acids can also damage cells within the colon $[9,10]$. The different physiological functions of bile acids and their implication in pathological processes highlight the importance of understanding circulating bile acid profiles in drug-induced hepatotoxicity. 
Acetaminophen (APAP) is a drug commonly used to relieve pain and decrease fever. When taken in therapeutics doses, APAP is considered a very safe drug. With excessive doses, APAP can become highly toxic [11]. In North America, it is the main cause of acute liver failure, and often requires liver transplantation if too severe or not treated rapidly enough [12]. In extreme cases, APAP toxicity can cause death within $48 \mathrm{~h}$. Previous studies have shown APAP interferes with bile acid synthesis [13-15].

Different LC-MS based methods to measure bile acids exist [16,17], but a gap still remains with regards to the wide range of bile acid derivatives that exist and their changing profiles with APAP dose. By studying the effect on individual bile acids, specific reactions related to bile acid metabolism can be assessed as being relevant to follow altered hepatic metabolism. The goal of this study was to develop an optimized and semi-quantitative method to evaluate the effects of APAP on numerous bile acids, including free and conjugated forms. Liquid chromatography coupled to tandem mass spectrometry is a powerful technique that offers many advantages for selective detection of individual bile acids, which are uniquely challenging due to the presence of many isomers. Bile acids can be difficult to analyze due to the similarities between the structures. In this study, we developed a rapid method to monitor 46 bile acids by LC-MS/MS on a triple quadrupole platform in multiple reaction monitoring (MRM) mode.

\section{Results and Discussion}

A targeted liquid chromatography-multiple reaction monitoring (LC-MRM) method was developed to monitor 46 bile acids in rat plasma following a simple sample preparation to evaluate the effect of increasing APAP dose. Bile acids were extracted by protein precipitation using methanol, following the addition of an isotopically labeled internal standard mix. A reverse-phase solid-core C18 column was employed to separate the 46 bile acids with excellent resolution and peak shape using acidified water and acetonitrile as mobile phase, within a 45 min gradient. As shown in Figure 1, all 46 bile acids in the standard mix were well resolved, including many bile acid isomers (e.g., UDCA, CDCA, and DCA). For example, LC-MRM chromatograms for $\alpha$-TMCA, $\beta$-TMCA, and TCA in rat plasma show good resolution obtained and highlight the usefulness of this method to monitor these isomers. The list of bile acids assessed in this study was based on the availability of a standard mix as well as multiple isotopically-labeled bile acids for relative quantitation, through a generous gift from MRM Proteomics Inc. The separation of these internal standard (IS) compounds is shown in Figure 2.

LC-MRM analyses in negative ion mode yielded better results than in positive ion mode in terms of sensitivity (data not shown), though both were optimized. In positive mode, precursor ions were often associated to in-source water losses and had limited sensitivity as compared to negative mode. In negative mode, unconjugated bile acids were monitored with two transitions, the highest signal coming from monitoring the pseudo-MRM transition of precursor ion to precursor ion, since their fragmentation resulted in a complex mix of fragments, thus limiting sensitivity for more specific fragment ions [18]. For conjugated bile acids, fragment ions resulting from the taurine and glycine moieties were employed as product ions. For each bile acid, however, secondary transitions were monitored for confirmatory purposes. In rat plasma samples, 39 of the 46 bile acids were measurable, with peaks having signal-to-noise of at least 10 and retention time matching that of the standard mix. No peak was observed for GDHCA, TDHCA, IDCA, DHCA, TLCA, AILCA and ILCA in rat plasma samples. DHCA is a synthetic product of the oxidation of CA and is mainly converted into 3- $\alpha$-hydroxylated-oxo bile acids [19]. It is therefore normal that the conjugated bile acids of DHCA (GDHCA and TDHCA) are not present in rat plasma either. Iso-bile acids (IDCA, AILCA and ILCA) are excreted in the feces of animals [20]. Of the 39 bile acids remaining, several had very small peaks that did not yield any statistically-significant changes between APAP doses, including GHCA, GLCA, GUDCA, NCA, NUDCA, DHLCA, LCA, di-oxo-LCA and 6,7 diketo-LCA. 

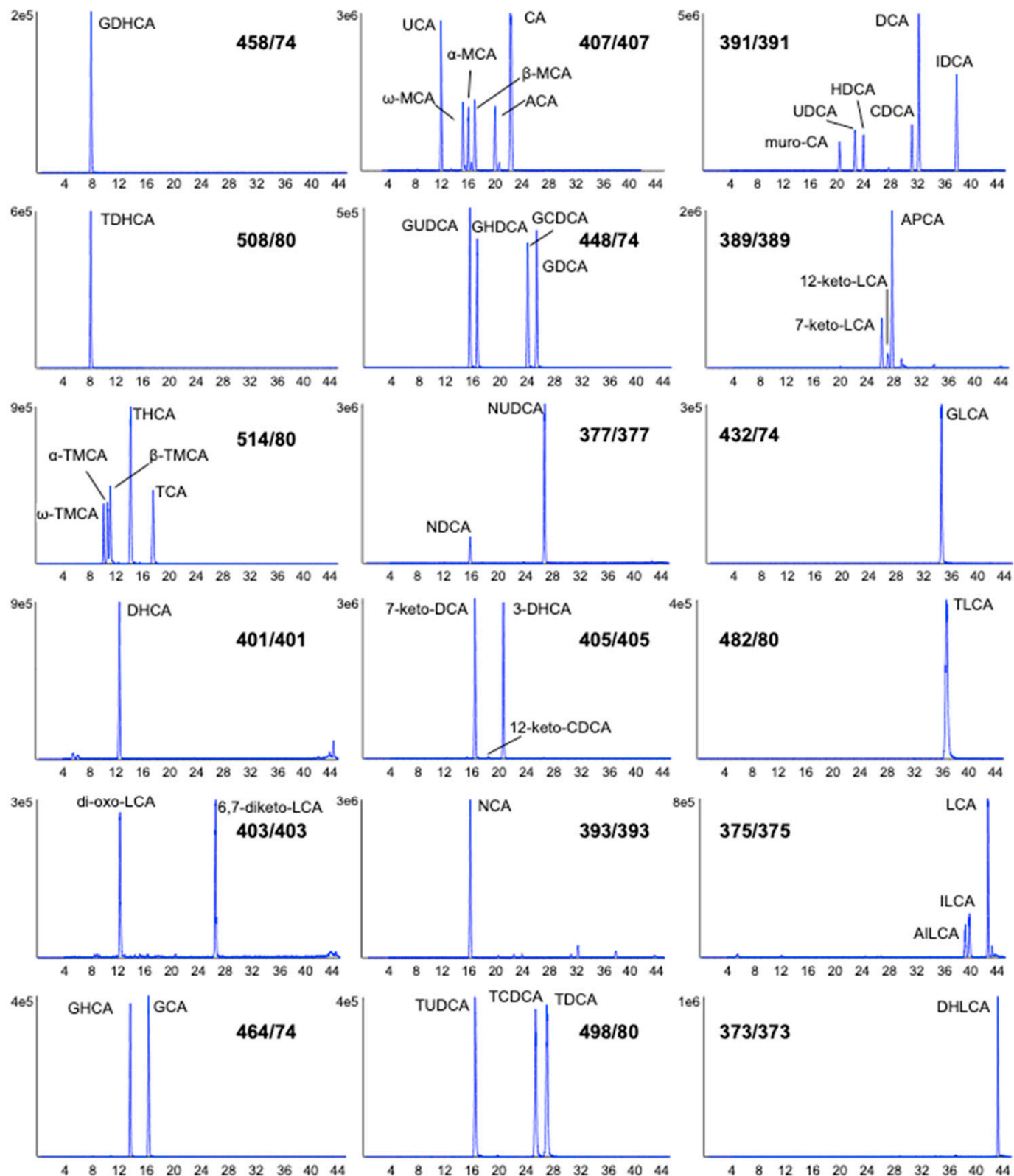

Figure 1. Representative LC-MRM chromatograms in negative mode of a standard mixture containing 46 bile acids (using the most sensitive transition for each bile acid, as shown). Acronyms for each bile acid species are listed in the Abbreviations (and Methods) section.

The highest APAP dose administered in this study significantly influenced the peaks corresponding to several bile acids (Figure 3). Table S1 shows the $p$-values and fold changes seen for each of these changing bile acids at each of the dosing levels compared to the lowest dose. This table also shows the integration data considering both MRM transitions monitored for each of these bile acids, and confirms that for all except two which were too small to properly integrate, these secondary transitions correlated well with the first (more sensitive) transition. Each MRM peak was also investigated for saturation effects. Although no linear ranges were determined directly, based on the peak heights of these bile acids, it was confirmed that we would be able to detect changes in terms of fold change (up or down). It is, however, very important to state here that fold changes of peak area ratios do not directly translate into concentration fold changes. These results are reported to determine which bile acids of the 46 from the standard mix were well observed in rat plasma samples and which were altered significantly with increased APAP dose. Thirteen bile acids of the 30 having significant signal-to-noise in our samples 
were shown to have statistically-relevant changes between the lowest and highest dose given in this study, with a $p$-value of lower than 0.05 , six of which had $p$-values lower than 0.01 . Increasing the APAP dose affected the concentration of some bile acids more than others. The bile acids with the most significant changes (with $p<0.01$ ) were GCA, GDCA, 7-keto-DCA, APCA, CA and DCA. The graphs in Figure 3 show the peak area ratios at all four doses of APAP. The taurine conjugates monitored did not show any statistically relevant changes with APAP dose. An important effect was seen, however, for several conjugated glycine conjugates. All four glycine conjugates having adequate peak size (GCA, GCDCA, GDCA and GHDCA) were found to significantly increase between 75 and $600 \mathrm{mg} / \mathrm{kg}$ APAP. The three with less obvious quantitative changes were notably much smaller peaks in the rat plasma extracts. For example, the peak area ratio for GDCA was 10.1 times higher (with a $p$-value of 0.0024 ) with $600 \mathrm{mg} / \mathrm{kg}$ compared with $75 \mathrm{mg} / \mathrm{kg}$ APAP, while the corresponding taurine conjugate, TDCA, did not show any effect at the highest dose. Since the conjugation of bile acids is an important pathway for their secretion by the liver, our results indicate that APAP could influence the transfer pathway of bile acids from the liver to the bloodstream.

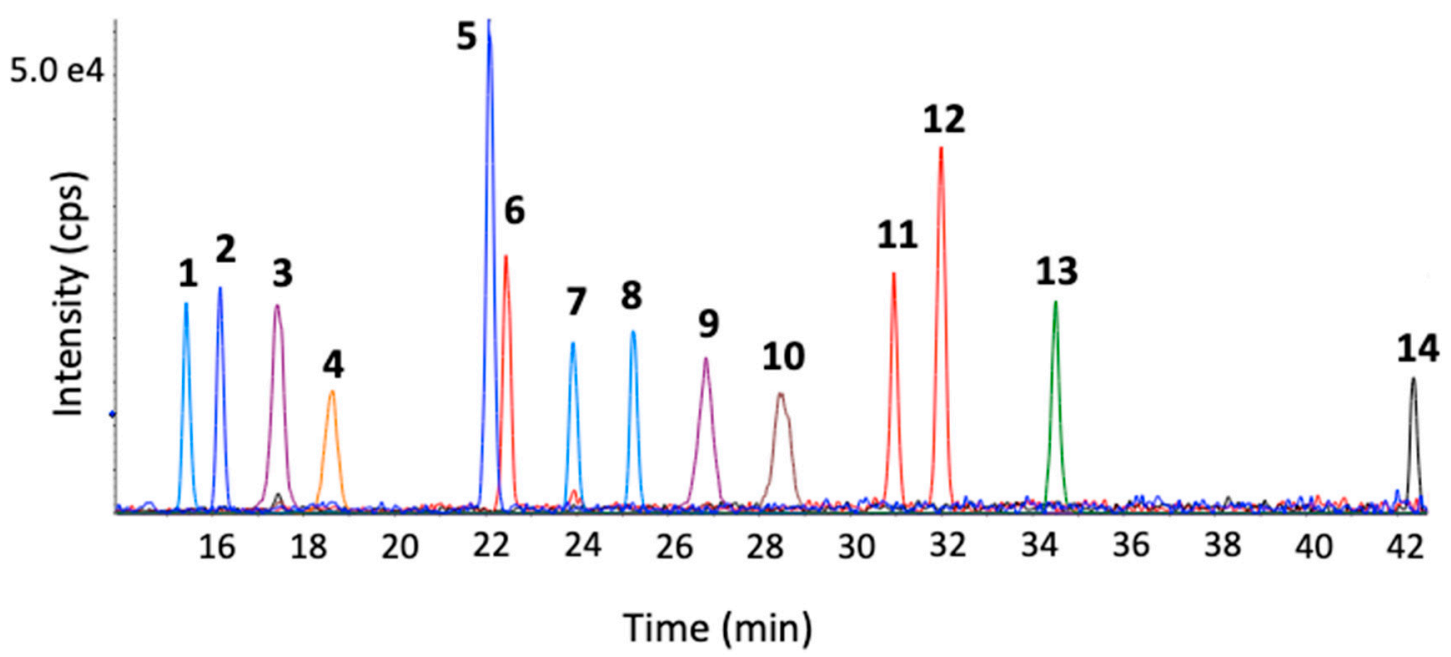

Figure 2. Representative LC-MRM chromatograms from a $20 \mu \mathrm{L}$ injection of IS mix containing 0.013-0.1 $\mu \mathrm{M}$ final concentration of each IS compound. (1) d4-GUDCA (2) d4-GCA (3) d4-TUDCA (4) d4-TCA (5) d4-CA (6) d4-UDCA (7) d4-GCDCA (8) d4-GDCA (9) d4-TCDCA (10) d6-TDCA (11) d4-CDCA (12) d4-DCA (13) d4-GLCA (14) d4-LCA.

We found that for the two primary bile acids, CDCA and CA, only CA was found to have a statistically significant increase with APAP dose levels (fold change of 1.8 and $p$-value of 0.005 at highest dose). Peak area ratios for $\alpha$-MCA, and $\omega$-MCA had increased by 4.6-, and 7.4-fold ( $p$-value of 0.0268 , and 0.0322), respectively. Given that CDCA is transformed by $6 \beta$-hydroxylase in rat liver into $\alpha$-MCA, $\beta-\mathrm{MCA}$, and $\omega-\mathrm{MCA}$, it is likely that CDCA is mostly converted into different MCA isomers [21]. The peak area ratio of DCA increased 5.6-fold, (with a $p$-value of 0.0003). Interestingly, DCA has been reported to induce both early apoptosis and necrosis, thus affecting cell development [22]. The fold changes between different individual bile acids cannot be directly compared, of course, since the relative response and sensitivity of each compound by LC-MS/MS is unique. We are not assuming that a larger fold change from this data set gives a stronger change in actual concentration. This would need a follow-up study for absolute quantitation of individual bile acids, with calibration curves for each. This is quite difficult, however, considering we are not able to construct traditional calibration curves for endogenous metabolites in complex biological matrices, such as plasma, as is done for therapeutic drug monitoring. 

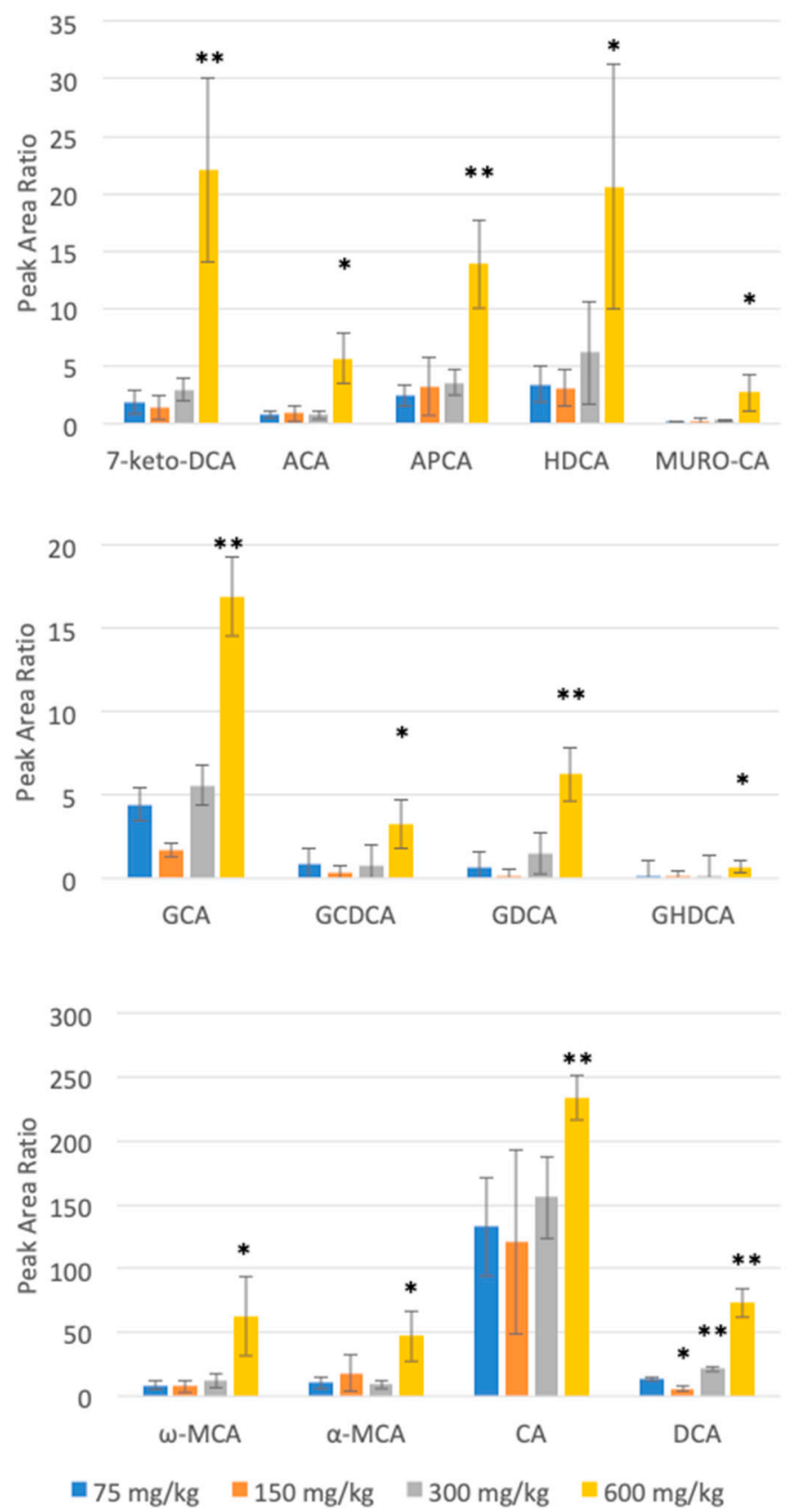

Figure 3. Peak area ratios for bile acids having significant changes between $75 \mathrm{mg} / \mathrm{kg}$ and $600 \mathrm{mg} / \mathrm{kg}$ APAP dosing, as measured in rat plasma after $24 \mathrm{~h} .{ }^{*} p$-value $<0.05,{ }^{* *} p$-value $<0.01$.

The LC-MRM data was imported into metabolomics software (MarkerView ${ }^{\mathrm{TM}}$ ) to perform statistical analyses (Student's t-test, as shown previously) and also to visualize data presented within a principal component analysis (PCA). Figure 4 shows the PCA plot of the first two principal components (PC1 vs. PC2), with Pareto scaling to alleviate bias to highest peaks. This plot shows clearly that the highest dose of $600 \mathrm{mg} / \mathrm{kg}$ clusters separately to the three lower doses $(75,150$, and $300 \mathrm{mg} / \mathrm{kg}$ ), as was evident from the t-testing results of the individual bile acids. The PCA plot, which used all features from the LC-MRM data, following supervised peak integration, serves to show that the high dose had a marked effect compared to the three lower doses, instead of seeing a gradual shift between the four doses. 


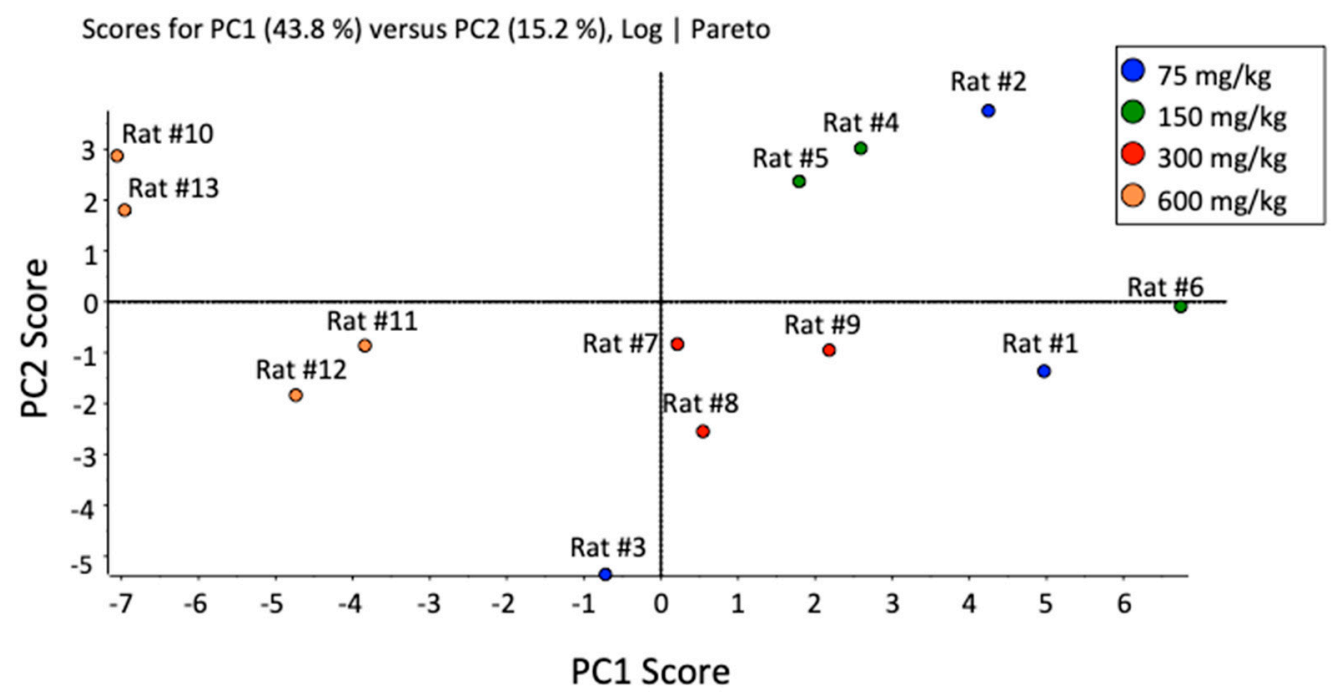

Figure 4. Unsupervised principal component analysis (PCA) of 39 bile acids detected in rat plasma samples (comparing four APAP dosing groups).

A higher throughput method could be devised to assess the specific bile acids perturbed by APAP in a follow-up study, for a more rapid assessment of changes in a clinical setting, for instance. It is important to note, however, that there exists many isomers of bile acids in biological samples and that even if we are interested in targeting a finite list of specific ones for a follow-up assay, we would still need to ensure proper separation of all these isomers. The study presented here focused specifically on evaluation the 46 bile acids available from a known standard mix. This method is not presented for the purpose of being a clinical assay, since it would likely not be high throughput enough considering the chromatographic separation needed to access all these different isomers. It also does not serve to accurately quantify each bile acid (in terms of concentration), rather it looks at relative amounts of bile acids (e.g., their profiles) in a biological matrix (rat plasma) to look for specific effects of APAP dose on individual bile acids. Therefore, this work should not be considered as a new validated method, as per US FDA guidelines. It would be interesting in a future study to validate a method for the bile acids specifically perturbed by high dose APAP. This is quite challenging in the case of endogenous metabolites since it would necessitate stable isotope standards for each metabolite to be quantified, as well as a suitable "blank" biological matrix to be used for preparing calibration curves for each analyte. Additionally, a non-targeted metabolomics approach using high-resolution tandem mass spectrometry would be able to access many more bile acid isomers, as well as sulfate and glucuronide metabolites, without the need for optimizing MS/MS parameters for MRM detection.

\section{Materials and Methods}

\subsection{Materials}

HPLC-grade acetonitrile (ACN) and methanol $(\mathrm{MeOH})$, as well as LC-MS-grade formic acid were purchased from Sigma-Aldrich (Oakville, ON, Canada). Purified water was prepared in-house. MetaboloMetrics ${ }^{\mathrm{TM}}$ bile acids analysis kits were obtained from MRM Proteomics Inc. (Montreal, QC, Canada). Kits contained a mix of 46 bile acids and an IS mix of 14 deuterated isotope-labeled internal standards. Sprague-Dawley rats were dosed (IP) with 75, 150, 300, and $600 \mathrm{mg} / \mathrm{kg}$ APAP, in triplicate, and plasma was collected after $24 \mathrm{~h}$ at INRS Centre de Biologie Experimentale (Laval, QC, Canada), within standard ethical practices. The protocol was approved by the Ethics Committee of the INRS Centre de Biologie Experimentale under the ethical practices of the Canadian Council on Animal Care (project UQLK.14.02). These samples were collected in February 2014 and stored at $-80{ }^{\circ} \mathrm{C}$ until proceeding with sample preparation. 
- The standard mix containing 46 bile acids (each at $2.5 \mathrm{nmol}$ ), except for deoxycholic acid $(5 \mathrm{nmol})$ and taurohyocholic acid $(6.5 \mathrm{nmol})$ was provided as a dried sample (Tube A). The bile acids in the standard mix were as follows: glycodehydrocholic acid (GDHCA), taurodehydrocholic acid (TDHCA), tauro- $\omega$-muricholic acid ( $\omega$-TMCA), tauro- $\alpha$-muricholic acid ( $\alpha$-TMCA), tauro- $\beta$-muricholic acid ( $\beta$-TMCA), taurohyocholic acid (THCA), taurocholic acid (TCA), dehydrocholic acid (DHCA), dioxolithocholic acid (di-oxo-LCA), 6,7-diketolithocholic acid (6,7-diketo-LCA), glycohyocholic acid (GHCA), glycocholic acid (GCA), ursocholic acid (UCA), $\omega$-muricholic acid ( $\omega$-MCA), $\alpha$-muricholic acid ( $\alpha$-MCA), $\beta$-muricholic acid ( $\beta$-MCA), allocholic acid (ACA), cholic acid (CA), glycoursodeoxycholic acid (GUDCA), glycohyodeoxycholic acid (GHDCA), glycochenodeoxycholic acid (GCDCA), glycodeoxycholic acid (GDCA), nordeoxycholic acid (NDCA), norursodeoxycholic acid (NUDCA), 7-ketodeoxycholic acid (7-keto-DCA), 12-ketodeoxycholic acid (12-keto-DCA), 3-dehydrocholic acid (3-DHCA), norcholic acid (NCA), tauroursodeoxycholic acid (TUDCA), taurochenodeoxycholic acid (TCDCA), taurodeoxycholic acid (TDCA), murocholic acid (muro-CA), ursodeoxycholic acid (UDCA), hyodeoxycholic acid (HDCA), chenodeoxycholic acid (CDCA), deoxycholic acid (DCA), isodeoxycholic acid (IDCA), 7-ketolithocholic acid (7-keto-LCA), 12-ketolithocholic acid (12-keto-LCA), apocholic acid (APCA), glycolithocholic acid (GLCA), taurolithocholic acid (TLCA), alloisolithocholic acid (AILCA), isolithocholic acid (ILCA), lithocholic acid (LCA) and dehydrolithocholic acid (DHLCA).

- Isotopically labeled bile acids were provided as an IS mix for normalization purposes. The labeled bile acids were present at between $0.1-0.75 \mathrm{nmol}$, as a dried sample (Tube B). The labeled bile acids in the IS mix were as follows: glycoursodeoxycholic acid- $\mathrm{d}_{4}\left(\mathrm{~d}_{4}\right.$-GUDCA), glycocholic acid- $\mathrm{d}_{4}\left(\mathrm{~d}_{4}-\mathrm{GCA}\right)$, tauroursodeoxycholic acid- $\mathrm{d}_{4}\left(\mathrm{~d}_{4}\right.$-TUDCA), taurocholic acid- $\mathrm{d}_{4}\left(\mathrm{~d}_{4}-\mathrm{TCA}\right)$, cholic acid- $\mathrm{d}_{4}\left(\mathrm{~d}_{4}-\mathrm{CA}\right)$, ursodeoxycholic acid- $\mathrm{d}_{4}\left(\mathrm{~d}_{4}\right.$-UDCA), glycochenodeoxycholic acid- $\mathrm{d}_{4}$ $\left(\mathrm{d}_{4}\right.$-GCDCA), glycodeoxycholic acid- $\mathrm{d}_{4}\left(\mathrm{~d}_{4}-5 \mathrm{GDCA}\right)$, taurochenodeoxycholic acid- $\mathrm{d}_{4}\left(\mathrm{~d}_{4}\right.$-TCDCA $)$, taurodeoxycholic acid- $\mathrm{d}_{6}\left(\mathrm{~d}_{6}-\mathrm{TDCA}\right)$, chenodeoxycholic acid- $\mathrm{d}_{4}\left(\mathrm{~d}_{4}-\mathrm{CDCA}\right)$, deoxycholic acid- $\mathrm{d}_{4}$ $\left(\mathrm{d}_{4}\right.$-DCA), glycolithocholic acid- $\mathrm{d}_{4}\left(\mathrm{~d}_{4}-\mathrm{GLCA}\right)$ and lithocholic acid- $\mathrm{d}_{4}\left(\mathrm{~d}_{4}-\mathrm{LCA}\right)$.

\subsection{Sample Preparation}

For the standard mix preparation, $250 \mu \mathrm{L}$ ACN was added to Tube A, followed by a 1:10 dilution with $40 \%$ ACN. This standard mix was injected $(2 \mu \mathrm{L})$ with the LC-MRM method described (shown in Figure 1) for retention time matching of bile acids in samples. For the internal standard (IS) mix, $7.5 \mathrm{~mL}$ of $40 \%$ ACN was added to Tube B. Plasma samples $(50 \mu \mathrm{L})$ were mixed with $50 \mu \mathrm{L}$ of the reconstituted (Tube B) internal standard solution. The extraction of bile acids from rat plasma was then performed by adding $300 \mu \mathrm{L} \mathrm{MeOH}$ to precipitate proteins. Samples were vortexed and sonicated for $15 \mathrm{~min}$, then centrifuged at 14,000 rpm for $8 \mathrm{~min}$. Supernatants $(300 \mu \mathrm{L})$ were transferred to new microtubes, dried under nitrogen and reconstituted with $150 \mu \mathrm{L} \mathrm{50 \%} \mathrm{MeOH}$ prior to analysis. Extracts were stored at $-20^{\circ} \mathrm{C}$ until LC-MS analysis.

\section{3. $L C-M S / M S$}

Extracted plasma and standards were separated on an Aeris ${ }^{\mathrm{TM}}$ PEPTIDE XB-C18 column $(1.7 \mu \mathrm{m}$, $100 \mathrm{~mm} \times 2.1 \mathrm{~mm})\left(\right.$ Phenomenex ${ }^{\circledR}$, Torrance, CA, USA) using a Nexera ultra high performance liquid chromatography (UHPLC) system (Shimadzu, Columbia, MD, USA) at $50{ }^{\circ} \mathrm{C}$ with gradient elution using water and $\mathrm{ACN}$, each containing $0.1 \%$ formic acid as mobile phase $\mathrm{A}$ and $\mathrm{B}$, respectively, at a flow rate of $0.400 \mathrm{~mL} / \mathrm{min}$ and injection volume of $15 \mu \mathrm{L}$. The gradient started at $10 \% \mathrm{~B}$ and was held for $1 \mathrm{~min}$ increased linearly to $25 \%$ for $2 \mathrm{~min}$, to $35 \%$ over $17 \mathrm{~min}$, to $50 \%$ over $20 \mathrm{~min}$, to $60 \%$ over $2 \mathrm{~min}$ and $90 \%$ for $1 \mathrm{~min}$, followed by a $10 \mathrm{~min}$ column re-equilibration time at starting conditions. MS data was collected using a QTRAP 5500 system (Sciex, Concord, ON, Canada). Electrospray ionization (ESI) in negative ion mode and multiple reaction monitoring (MRM) was used. The MRM parameters (first and second transitions with collision energies (CE)) for all 46 bile acids are listed in Table 1). Each transition was monitored throughout the chromatogram with a dwell time of $7 \mathrm{~ms}$. 
Because of the fragmentation behavior of the deprotonated unconjugated bile acids, the first transitions chosen for the unconjugated forms were simply precursor to precursor ions, as has been done in many previous reports on bile acid analysis [17,23-25]. This was necessary for ensuring the best sensitivity of detection for these unconjugated forms. For glycine conjugated bile acids, there was a specific and sensitive common fragment ion at $m / z 74$, corresponding to the deprotonated glycine moiety being lost. Similarly, taurine conjugates yielded a common fragment at $m / z 80$, corresponding to the $\mathrm{HSO}_{3}{ }^{-}$ ion from the taurine group. The secondary transitions were used for confirmation. For those bile acids found to be altered significantly upon APAP dose, the secondary transition was also integrated and compared.

Table 1. Optimized MRM transitions and collision energies for 46 standard bile acids along with their respective retention times.

\begin{tabular}{|c|c|c|c|c|}
\hline Bile Acid & RT (min) & 1st Transition (CE) & 2nd Transition (CE) & IS \\
\hline GDHCA & 7.9 & $458.2 / 74.0(-66)$ & $458.2 / 348.1(-41)$ & CDCA-d 4 \\
\hline TDHCA & 8.2 & $508.2 / 80.0(-123)$ & $508.2 / 124.1(-67)$ & CDCA- $\mathrm{d}_{4}$ \\
\hline T- $\omega-\mathrm{MCA}$ & 10.1 & $514.2 / 80.0(-135)$ & $514.2 / 107.0(-82)$ & TCA- $\mathrm{d}_{4}$ \\
\hline $\mathrm{T}-\alpha-\mathrm{MCA}$ & 10.7 & $514.2 / 80.0(-135)$ & $514.2 / 107.0(-82)$ & TCA-d 4 \\
\hline T- $\beta-\mathrm{MCA}$ & 11.1 & $514.2 / 80.0(-135)$ & $514.2 / 124.0(-65)$ & TCA-d 4 \\
\hline UCA & 11.9 & $407.2 / 407.2(-15)$ & $407.2 / 343.1(-46)$ & CA- $\mathrm{d}_{4}$ \\
\hline di-oxo-LCA & 12.3 & 403.2/403.2 (-18) & $403.2 / 385.2(-40)$ & CDCA-d 4 \\
\hline DHCA & 12.4 & $401.2 / 401.2(-18)$ & $401.2 / 331.1(-36)$ & CDCA- $\mathrm{d}_{4}$ \\
\hline GHCA & 13.6 & $464.2 / 74.0(-82)$ & $464.2 / 354.1(-56)$ & GCA-d 4 \\
\hline THCA & 14.1 & $514.2 / 80.0(-135)$ & $514.2 / 107.0(-82)$ & TCA-d 4 \\
\hline$\omega-\mathrm{MCA}$ & 15.1 & $407.2 / 407.2(-15)$ & $407.2 / 371.1(-43)$ & CA- $\mathrm{d}_{4}$ \\
\hline GUDCA & 15.5 & $448.2 / 74.0(-83)$ & $448.2 / 386.1(-59)$ & GUDCA-d $\mathrm{d}_{4}$ \\
\hline NDCA & 15.8 & $377.2 / 377.2(-15)$ & $377.2 / 331.1(-46)$ & CDCA- $\mathrm{d}_{4}$ \\
\hline$\alpha-\mathrm{MCA}$ & 16.0 & $407.2 / 407.2(-15)$ & $407.2 / 371.1(-43)$ & CA- $\mathrm{d}_{4}$ \\
\hline NCA & 16.1 & 393.2/393.2 (-15) & $393.2 / 375.1(-45)$ & CDCA-d 4 \\
\hline GCA & 16.2 & $464.2 / 74.0(-82)$ & $464.2 / 402.1(-46)$ & GCA-d 4 \\
\hline 7-keto-DCA & 16.4 & $405.2 / 405.2(-18)$ & $405.2 / 289.1(-51)$ & CDCA-d 4 \\
\hline TUDCA & 16.4 & $498.2 / 80.0(-130)$ & $498.2 / 107(-82)$ & TUDCA-d 4 \\
\hline GHDCA & 16.6 & $448.2 / 74.0(-83)$ & $448.2 / 386.1(-59)$ & GUDCA- $\mathrm{d}_{4}$ \\
\hline$\beta$-MCA & 16.9 & $407.2 / 407.2(-15)$ & $407.2 / 371.1(-43)$ & CA-d 4 \\
\hline TCA & 17.5 & $514.2 / 80.0(-135)$ & $514.2 / 124.0(-65)$ & TCA- $\mathrm{d}_{4}$ \\
\hline 12-keto-CDCA & 18.4 & $405.2 / 405.2(-18)$ & $405.2 / 387.1(-45)$ & CDCA-d 4 \\
\hline ACA & 19.9 & $407.2 / 407.2(-15)$ & $407.2 / 371.1(-43)$ & CA-d 4 \\
\hline muro-CA & 20.3 & $391.2 / 391.2(-15)$ & $391.2 / 343.1(-53)$ & UDCA- $\mathrm{d}_{4}$ \\
\hline 3-DHCA & 20.5 & $405.2 / 405.2(-18)$ & $405.2 / 289.1(-51)$ & CDCA-d 4 \\
\hline CA & 22.2 & $407.2 / 407.2(-15)$ & $407.2 / 343.1(-46)$ & $\mathrm{CA}-\mathrm{d}_{4}$ \\
\hline UDCA & 22.6 & $391.2 / 391.2(-15)$ & $391.2 / 373.2(-48)$ & UDCA- $\mathrm{d}_{4}$ \\
\hline HDCA & 23.9 & $391.2 / 391.2(-15)$ & $391.2 / 373.2(-48)$ & CDCA- $\mathrm{d}_{4}$ \\
\hline GCDCA & 24.0 & $448.2 / 74.0(-83)$ & $448.2 / 404.2(-46)$ & GCDCA-d ${ }_{4}$ \\
\hline TCDCA & 25.3 & $498.2 / 80.0(-130)$ & $498.2 / 124(-64)$ & TCDCA- $\mathrm{d}_{4}$ \\
\hline
\end{tabular}


Table 1. Cont.

\begin{tabular}{ccccc}
\hline Bile Acid & RT (min) & 1st Transition (CE) & 2nd Transition (CE) & IS \\
\hline GDCA & 25.3 & $448.2 / 74.0(-83)$ & $448.2 / 404.2(-46)$ & GDCA-d $_{4}$ \\
\hline 7-keto-LCA & 26.1 & $389.2 / 389.2(-18)$ & $389.2 / 354.1(-43)$ & LCA-d $_{4}$ \\
\hline 6,7-diketo-LCA & 26.5 & $403.2 / 403.2(-18)$ & $403.2 / 347.1(-39)$ & LCA-d $_{4}$ \\
\hline NUDCA & 26.7 & $377.2 / 377.2(-15)$ & $377.2 / 359.1(-45)$ & CDCA-d $_{4}$ \\
\hline TDCA & 27.0 & $498.2 / 80.0(-130)$ & $498.2 / 124(-64)$ & TDCA-d $_{6}$ \\
\hline 12-keto-LCA & 27.1 & $389.2 / 389.2(-18)$ & $389.2 / 354.1(-43)$ & LCA-d $_{4}$ \\
\hline APCA & 27.6 & $389.2 / 389.2(-18)$ & $389.2 / 371.1(-43)$ & CDCA-d $_{4}$ \\
\hline CDCA & 31.1 & $391.2 / 391.2(-15)$ & $391.2 / 373.2(-48)$ & CDCA-d \\
\hline DCA & 32.1 & $391.2 / 391.2(-15)$ & $391.2 / 343.1(-53)$ & DCA-d $_{4}$ \\
\hline GLCA & 34.6 & $432.2 / 74.0(-66)$ & $432.2 / 388.1(-45)$ & GLCA-d $_{4}$ \\
\hline TLCA & 36.6 & $482.2 / 80.0(-135)$ & $482.2 / 107(-80)$ & LCA-d $_{4}$ \\
\hline IDCA & 37.8 & $391.2 / 391.2(-15)$ & $391.2 / 345.1(-45)$ & DCA-d $_{4}$ \\
\hline AILCA & 39.1 & $375.2 / 375.2(-15)$ & $375.2 / 45(-50)$ & LCA-d $_{4}$ \\
\hline ILCA & 39.7 & $375.2 / 375.2(-15)$ & $375.2 / 45(-50)$ & LCA-d $_{4}$ \\
\hline LCA & 42.5 & $375.2 / 375.2(-15)$ & $375.2 / 45(-50)$ & LCA-d $_{4}$ \\
\hline DHLCA & 43.1 & $373.2 / 373.2(-18)$ & $373.2 / 45(-50)$ & LCA-d $_{4}$ \\
\hline
\end{tabular}

\subsection{Statistical Analysis}

A mixture of 14 deuterated bile acids was added to the plasma samples prior to metabolite extraction, for normalization of data as peak area ratios (analyte/IS) (see Table 2). For those bile acids without corresponding deuterated analogs, the closest eluting deuterated analog was used as IS, as noted in Table 1. Standards were used to confirm the identity of each bile acid, based on retention and MRM signal. Peak integration was performed using Multiquant ${ }^{\text {TM }} 2.1$ (Sciex). Statistical analyses were done using MarkerView ${ }^{\mathrm{TM}}$ 1.2.1 (Sciex). This software was used to perform Student's t-tests, yielding $p$-values and fold changes between different dosing groups, for each bile acid detected in rat plasma samples. Within Markerview software, principal component analysis was performed on the integrated LC-MRM data, without weighting and using Pareto scaling (unsupervised).

Table 2. Optimized MRM transitions and collision energies for 14 internal standards compounds, along with their respective retention times.

\begin{tabular}{ccccc}
\hline & RT (min) & Q1 $(m / z)$ & Q3 $(m / z)$ & CE (V) \\
\hline GUDCA-d $_{4}$ & 15.5 & 452.3 & 74.0 & -41 \\
GCA-d $_{4}$ & 16.2 & 468.3 & 74.0 & -45 \\
TUDCA-d $_{4}$ & 17.4 & 502.3 & 80.0 & -73 \\
TCA-d $_{4}$ & 18.7 & 518.3 & 80.0 & -80 \\
CA-d & 22.1 & 411.3 & 411.3 & -15 \\
UDCA-d & 22.5 & 395.3 & 395.3 & -15 \\
GCDCA-d $_{4}$ & 23.8 & 452.3 & 74.0 & -37 \\
GDCA-d & 25.3 & 452.3 & 74.0 & -41 \\
TCDCA-d $_{4}$ & 26.8 & 502.3 & 80.0 & -80 \\
TDCA-d & 28.7 & 504.3 & 80.0 & -80 \\
CDCA-d & 31 & 395.3 & 395.3 & -15 \\
DCA-d & 32 & 395.3 & 395.3 & -15 \\
GLCA-d & 34.5 & 436.3 & 74.0 & -41 \\
LCA-d $_{4}$ & 42.4 & 379.3 & 379.3 & -15 \\
\hline
\end{tabular}




\section{Conclusions}

In this study, we have developed a targeted metabolomics method to gain a better understanding of the effects of APAP on circulating bile acid profiles. A simple protein precipitation procedure in rat plasma was employed rapidly prepare samples for analysis. A standard mix of 46 bile acids was successfully resolved by LC-MRM, 39 of which were detected in rat plasma samples. These analyses highlighted significant changes in bile acid profiles with increasing APAP dose in rats. In general, these results indicate that APAP can have an important effect on the metabolism of bile acids. Depending on the dose level, exposure to high or repeated APAP doses has the potential to induce serious health problems, related to bile acid metabolism and excretion. The specificity of these biomarkers to APAP-related toxicity would still need to be investigated. Certain of these bile acids can also serve as biomarkers to establish the level of hepatotoxicity; however, more work would be needed to validate specific bile acid biomarkers for clinical use.

Supplementary Materials: The following are available online at http://www.mdpi.com/2218-1989/10/1/26/s1, Table S1: Comparison of $p$-values and fold-changes between the four doses of APAP administered in this study (using two MRM transitions for each peak) of known bile acids showing significant differences between the lowest and highest dose APAP.

Author Contributions: Conceptualization, L.S.; methodology, V.P., L.O., L.S.; formal analysis, V.P.; data curation, V.P.; writing—original draft preparation, V.P., L.S.; writing-review and editing, L.S.; supervision, L.S.; funding acquisition, L.S. All authors have read and agreed to the published version of the manuscript.

Funding: This research was funded by the Natural Sciences and Engineering Research Council of Canada (NSERC), discovery grant program (grant number 355933-2016).

Acknowledgments: We would like to thank MRM Proteomics Inc. for the generous gift of the bile acid kit, containing the standard and IS mix.

Conflicts of Interest: The authors declare no conflict of interest. The funders had no role in the design of the study; in the collection, analyses, or interpretation of data; in the writing of the manuscript, or in the decision to publish the results.

$\begin{array}{ll}\text { Abbreviations } & \\ \text { GDHCA } & \text { glycodehydrocholic acid } \\ \text { TDHCA } & \text { taurodehydrocholic acid } \\ \omega \text {-TMCA } & \text { tauro- } \omega \text {-muricholic acid } \\ \alpha \text {-TMCA } & \text { tauro- } \alpha \text {-muricholic acid } \\ \beta \text {-TMCA } & \text { tauro- } \beta \text {-muricholic acid } \\ \text { THCA } & \text { taurohyocholic acid } \\ \text { TCA } & \text { taurocholic acid } \\ \text { DHCA } & \text { dehydrocholic acid } \\ \text { di-oxo-LCA } & \text { dioxolithocholic acid } \\ 6,7-\text { diketo-LCA } & 6,7-\text { diketolithocholic acid } \\ \text { GHCA } & \text { glycohyocholic acid } \\ \text { GCA } & \text { glycocholic acid } \\ \text { UCA } & \text { ursocholic acid } \\ \omega \text {-MCA } & \omega \text {-muricholic acid } \\ \alpha \text {-MCA } & \alpha \text {-muricholic acid } \\ \beta \text {-MCA } & \beta \text {-muricholic acid } \\ \text { ACA } & \text { allocholic acid } \\ \text { CA } & \text { cholic acid } \\ \text { GUDCA } & \text { glycoursodeoxycholic acid } \\ \text { GHDCA } & \text { glycohyodeoxycholic acid } \\ \text { GCDCA } & \text { glycochenodeoxycholic acid } \\ \text { GDCA } & \text { glycodeoxycholic acid } \\ \text { NDCA } & \text { nordeoxycholic acid } \\ \text { NUDCA } & \text { norursodeoxycholic acid } \\ & \end{array}$




$\begin{array}{ll}\text { 7-keto-DCA } & \text { 7-ketodeoxycholic acid } \\ \text { 12-keto-DCA } & \text { 12-ketodeoxycholic acid } \\ \text { 3-DHCA } & \text { 3-dehydrocholic acid } \\ \text { NCA } & \text { norcholic acid } \\ \text { TUDCA } & \text { tauroursodeoxycholic acid } \\ \text { TCDCA } & \text { taurochenodeoxycholic acid } \\ \text { TDCA } & \text { taurodeoxycholic acid } \\ \text { muro-CA } & \text { murocholic acid } \\ \text { UDCA } & \text { ursodeoxycholic acid } \\ \text { HDCA } & \text { hyodeoxycholic acid } \\ \text { CDCA } & \text { chenodeoxycholic acid } \\ \text { DCA } & \text { deoxycholic acid } \\ \text { IDCA } & \text { isodeoxycholic acid } \\ \text { 7-keto-LCA } & \text { 7-ketolithocholic acid } \\ \text { 12-keto-LCA } & \text { 12-ketolithocholic acid } \\ \text { APCA } & \text { apocholic acid } \\ \text { GLCA } & \text { glycolithocholic acid } \\ \text { TLCA } & \text { taurolithocholic acid } \\ \text { AILCA } & \text { alloisolithocholic acid } \\ \text { ILCA } & \text { isolithocholic acid } \\ \text { LCA } & \text { lithocholic acid } \\ \text { DHLCA } & \text { dehydrolithocholic acid } \\ & \end{array}$

\section{References}

1. Russell, D.W. Fifty years of advances in bile acid synthesis and metabolism. J. Lipid Res. 2009, 50, S120-S125. [CrossRef] [PubMed]

2. Wilson, J.D. The role of bile acids in the overall regulation of steroid metabolism. Arch. Intern. Med. 1972, 130, 493-505. [CrossRef]

3. Boyer, J.L. Bile formation and secretion. Compr. Physiol. 2013, 3, 1035-1078. [CrossRef]

4. Hofmann, A.F. Bile Acids: The Good, the Bad, and the Ugly. News Physiol. Sci. 1999, 14, 24-29. [CrossRef] [PubMed]

5. Copple, B.L.; Jaeschke, H.; Klaassen, C.D. Oxidative stress and the pathogenesis of cholestasis. Semin. Liver Dis. 2010, 30, 195-204. [CrossRef] [PubMed]

6. Fang, Y.; Han, S.I.; Mitchell, C.; Gupta, S.; Studer, E.; Grant, S.; Hylemon, P.B.; Dent, P. Bile acids induce mitochondrial ROS, which promote activation of receptor tyrosine kinases and signaling pathways in rat hepatocytes. Hepatology 2004, 40, 961-971. [CrossRef] [PubMed]

7. Gupta, S.; Natarajan, R.; Payne, S.G.; Studer, E.J.; Spiegel, S.; Dent, P.; Hylemon, P.B. Deoxycholic acid activates the c-Jun N-terminal kinase pathway via FAS receptor activation in primary hepatocytes: Role of acidic sphingomyelinase-mediated ceramide generation in FAS receptor activation. J. Biol. Chem. 2004, 279, 5821-5828. [CrossRef] [PubMed]

8. Jaeschke, H.; Gores, G.J.; Cederbaum, A.I.; Hinson, J.A.; Pessayre, D.; Lemasters, J.J. Mechanisms of hepatotoxicity. Toxicol. Sci. 2002, 65, 166-176. [CrossRef]

9. Reddy, B.S.; Weisburger, J.H.; Wynder, E.L. Effects of high risk and low risk diets for colon carcinogenesis on fecal microflora and steroids in man. J. Nutr. 1975, 105, 878-884. [CrossRef]

10. Nagengast, F.M.; Grubben, M.J.A.L.; van Munster, I.P. Role of bile acids in colorectal carcinogenesis. Eur. J. Cancer 1995, 31, 1067-1070. [CrossRef]

11. Larson, A.M.; Polson, J.; Fontana, R.J.; Davern, T.J.; Lalani, E.; Hynan, L.S.; Reisch, J.S.; SchiØdt, F.V.; Ostapowicz, G.; Shakil, A.O.; et al. Acetaminophen-induced acute liver failure: Results of a United States multicenter, prospective study. Hepatology 2005, 42, 1364-1372. [CrossRef] [PubMed]

12. Lee, W.M. Acute liver failure in the United States. Semin. Liver Dis. 2003, 23, 217-226. [CrossRef]

13. Woolbright, B.L.; McGill, M.R.; Staggs, V.S.; Winefield, R.D.; Gholami, P.; Olyaee, M.; Sharpe, M.R.; Curry, S.C.; Lee, W.M.; Jaeschke, H.; et al. Glycodeoxycholic acid levels as prognostic biomarker in acetaminophen-induced acute liver failure patients. Toxicol. Sci. 2014, 142, 436-444. [CrossRef] 
14. James, L.; Yan, K.; Pence, L.; Simpson, P.; Bhattacharyya, S.; Gill, P.; Letzig, L.; Kearns, G.; Beger, R. Comparison of Bile Acids and Acetaminophen Protein Adducts in Children and Adolescents with Acetaminophen Toxicity. PLoS ONE 2015, 10, e0131010. [CrossRef]

15. Bhushan, B.; Borude, P.; Edwards, G.; Walesky, C.; Cleveland, J.; Li, F.; Ma, X.; Apte, U. Role of bile acids in liver injury and regeneration following acetaminophen overdose. Am. J. Pathol. 2013, 183, 1518-1526. [CrossRef]

16. Scherer, M.; Gnewuch, C.; Schmitz, G.; Liebisch, G. Rapid quantification of bile acids and their conjugates in serum by liquid chromatography-tandem mass spectrometry. J. Chromatogr. B 2009, 877, 3920-3925. [CrossRef]

17. Suzuki, Y.; Kaneko, R.; Nomura, M.; Naito, H.; Kitamori, K.; Nakajima, T.; Ogawa, T.; Hattori, H.; Seno, H.; Ishii, A. Simple and rapid quantitation of 21 bile acids in rat serum and liver by UPLC-MS-MS: Effect of high fat diet on glycine conjugates of rat bile acids. Nagoya J. Med. Sci. 2013, 75, 57-72. [CrossRef]

18. Han, J.; Liu, Y.; Wang, R.; Yang, J.; Ling, V.; Borchers, C.H. Metabolic profiling of bile acids in human and mouse blood by LC-MS/MS in combination with phospholipid-depletion solid-phase extraction. Anal. Chem. 2015, 87, 1127-1136. [CrossRef]

19. Yousef, I.M.; Mignault, D.; Weber, A.M.; Tuchweber, B. Influence of dehydrocholic acid on the secretion of bile acids and biliary lipids in rats. Digestion 1990, 45, 40-51. [CrossRef] [PubMed]

20. Shefe, S.; Salen, G.; Hauser, S.; Dayal, B.; Batta, A.K. Metabolism of Iso-Bile Acids in the Rat. J. Biol. Chem. 1982, 257, 1401-1406.

21. Voigt, W.; Thomas, P.J.; Hsia, S.L. Enzymatic studies of bile acid metabolism. I. 6Beta-hydroxylation of chenodeoxycholic and taurochenodeoxycholic acids by microsomal preparations of rat liver. J. Biol. Chem. 1968, 243, 3493-3499. [PubMed]

22. Shiraki, K.; Ito, T.; Sugimoto, K.; Fuke, H.; Inoue, T.; Miyashita, K.; Yamanaka, T.; Suzuki, M.; Nabeshima, K.; Nakano, T.; et al. Different effects of bile acids, ursodeoxycholic acid and deoxycholic acid, on cell growth and cell death in human colonic adenocarcinoma cells. Int. J. Mol. Med. 2005, 16, 729-733. [PubMed]

23. Cai, X.; Liu, Y.; Zhou, X.; Navaneethan, U.; Shen, B.; Guo, B. An LC-ESI-MS method for the quantitative analysis of bile acids composition in fecal materials. Biomed. Chromatogr. 2012, 26, 101-108. [CrossRef] [PubMed]

24. Tagliacozzi, D.; Mozzi, A.F.; Casetta, B.; Bertucci, P.; Bernardini, S.; Di Ilio, C.; Urbani, A.; Federici, G. Quantitative analysis of bile acids in human plasma by liquid chromatography-electrospray tandem mass spectrometry: A simple and rapid one-step method. Clin. Chem. Lab. Med. 2003, 41, 1633-1641. [CrossRef]

25. Garcia-Canaveras, J.C.; Donato, M.T.; Castell, J.V.; Lahoz, A. Targeted profiling of circulating and hepatic bile acids in human, mouse, and rat using a UPLC-MRM-MS-validated method. J. Lipid Res. 2012, 53, 2231-2241. [CrossRef]

(C) 2020 by the authors. Licensee MDPI, Basel, Switzerland. This article is an open access article distributed under the terms and conditions of the Creative Commons Attribution (CC BY) license (http://creativecommons.org/licenses/by/4.0/). 